VOL. 67 (2003) [67-77]

\title{
ON D-KKM THEOREM AND ITS APPLICATIONS
}

\author{
H.K. Pathak AND M.S. Khan
}

In this paper, we introduce a new class of set-valued mappings in a non-convex setting called D-KKM mappings and prove a general D-KKM theorem. This extends and improves the KKM theorem for several families of set-valued mappings, such as $\mathbb{M}(X, Y), \mathbb{K}_{C}(X, Y), \mathbb{V}_{C}(X, Y), \mathbb{A}_{C}(X, Y)$ and $\mathfrak{U}_{C}(X, Y)$. In the sequel, we apply our theorem to get some existence results for maximal elements, generalised variational inequalities, and price equilibria.

\section{INTRODUCTION}

The celebrated Kanaster-Kuratoski-Mazurkiewicz Theorem [6] (KKM Theorem) and Fan's Lemma [4] are the fundamental works on which the whole KKM theory of nonlinear analysis rests. In the last two decades tremendous progress has been made in the theory and applicatons of the KKM Theorem and Fan's Lemma (see, for instance, $[7,8,10,11,12])$.

Let $E$ be a topological vector space and $X$ be a convex subset of $E$. A set valued mapping $T: X \rightarrow 2^{E}$ is called a KKM mapping if for each finite subset $\left\{x_{1}, x_{2}, \ldots, x_{n}\right\}$ of $X$,

$$
\operatorname{co}\left\{x_{1}, x_{2}, \ldots, x_{n}\right\} \subseteq \bigcup_{i=1}^{n} T x_{i}
$$

It is well known (refer to $[6,10]$ ) that if $T$ is a KKM mapping with closed values, then the family $\{T x: x \in X\}$ has the finite intersection property.

There is an interesting generalisaton of the above results (see, for instance [5, $\mathbf{6 , 8}, \mathbf{9}, \mathbf{1 0}])$ to the following.

Let $X$ be a convex space, $Y$ a topological space, and let $S, T: X \rightarrow 2^{Y}$ be two set-valued mappings such that for each finite subset $\left\{x_{1}, x_{2}, \ldots, x_{n}\right\}$ of $X$,

$$
T\left(\operatorname{co}\left\{x_{1}, x_{2}, \ldots, x_{n}\right\}\right) \subseteq \bigcup_{i=1}^{n} S x_{i} \quad \text { (KKM property) }
$$

Then the family $\{S x: x \in X\}$ has the finite intersection property under some conditions on $T$.

Received 16th April, 2002

Copyright Clearance Centre, Inc. Serial-fee code: 0004-9727/03 \$A2.00+0.00. 
In this paper, motivated by the above results, we introduce a new family of set valued mappings called D-KKM mappings and prove a general D-KKM Theorem. As applications of this theorem we obtain some results concerning the existence of maximal elements, generalised variational inequalities, and price equilibrium.

\section{Preliminaries:}

A multifunction (or set-valued mapping) $F: X \rightarrow 2^{Y}$ is a function from a set $X$ into the set $2^{Y}$ of nonempty subsets of a set $Y$.

For topological spaces $X$ and $Y$, a multifunction $F: X \rightarrow 2^{Y}$ is said to be upper semicontinuous if for each closed subset $B$ of $Y,\{x \in X: F x \cap B \neq \phi\}$ is closed, $F$ is closed if $F$ is closed in $X \times Y$, and compact if the range $F(X)$ is contained in a compact subset of $Y$.

Recall that a nonempty topological space is acyclic if all of its reduced Cech homology groups over rationals vanish.

Let $X$ and $Y$ be two sets and $D$ a non-empty subset of $X$ and denote $2^{Y}=\{B$ $\subseteq Y: B \neq \phi\}$, and let $T: X \rightarrow 2^{Y}$ be a set-valued mapping. In the sequel, we shall use the following notations:

(i) $T A=\bigcup_{x \in A} T x$ for $Z \subseteq X$;

(ii) $T_{D}^{-1} y=\{x \in D: y \in T x\}$; and $T_{D}^{*} y=\{x \in D: y \notin T x\}$ for each $y \in Y$.

Let $X$ be a set (in a vector space $E$ ) and $D$ a nonempty subset of $X$, and let $\langle D\rangle$ denote the set of nonempty finite subsets of $D$. Then $(X, D)$ is called a convex space if convex hulls of any $A \in\langle D\rangle$ is contained in $D$ and $X$ has a topology that induces the Euclidean topology on co $A$, where co denotes the convex hull. Such a convex hull will be called a polytope of $(X, D)$. Let $k_{C}(E)$ denote the set of nonempty compact convex subsets of $E$.

Let $\mathcal{T}$ be the class of set-valued mappings. Denote

$$
\begin{aligned}
\mathfrak{I}(X, Y) & =\left\{T: X \rightarrow 2^{Y} \mid T \in \mathfrak{I}\right\} \text { and } \\
\mathfrak{I}_{C} & =\left\{T_{n}, T_{n-1}, \ldots, T_{1} \mid T_{i} \in \mathfrak{I}, I=1,2, \ldots, n \text { for some } n\right\}
\end{aligned}
$$

that is, the set of finite composites of mappings in $\mathfrak{I}$.

For topological spaces $X$ and $Y$, we define

$\mathbb{C}(X, Y)=\{s: X \rightarrow Y \mid s$ is a single-valued continuous function $\}$;

$\mathbb{K}(X, Y)=\left\{T: X \rightarrow 2^{Y} \mid T\right.$ is a Kakutani map; that is $Y$ is a convex space and $T$ is upper semicontinuous with $T x \in k_{C}(Y)$ for $x \in X$; ;

$\mathrm{V}(X, Y)=\left\{T: X \rightarrow 2^{Y} \mid T\right.$ is an acyclic map; that is, $T$ is upper semicontinuous with compact acyclic values $\}$; 
$\mathbf{A}(X, Y)=\left\{T: X \rightarrow 2^{Y} \mid T\right.$ is a upper semicontinuous and approachable with compact values $\}$ (see [1]); and

$\mathbb{M}(X, Y)=\left\{T: X \rightarrow 2^{Y} \mid T\right.$ is an Aronszajn map with $R_{\delta}$ values $\}$.

A subset $A$ of $(X, D)$ is said to be D-convex if, for any $N \in\langle D\rangle, N \subseteq A$ implies co $N \subseteq A$. If $X=D$, then $X=(X, X)$ becomes a convex space in the sense of Lassonde [5].

The following abstract class of set-valued mappings was introduced by Park [8].

A class of $\mathfrak{U}$ of maps is defined by the following properties:

(i) $\mathfrak{U}$ contains the class $\mathbb{C}$ of (single-valued) continuous functions;

(ii) each $F \in \mathfrak{U}_{C}$ is upper semicontinuous and compact-valued; and

(iii) for any polytope $P, F \in \mathfrak{U}_{C}(P, P)$ has a fixed point.

Examples of $\mathfrak{U}$ are $\mathbb{C}$, the Kakutani maps $\mathbb{K}$ (with convex values), the Aronszajn maps $\mathbb{M}$ (with $R_{\delta}$ values, $R_{\delta}$ sets $\left\{X=\cap X_{i}: X_{i+1} \subseteq X_{i}, X_{i} \in A R\right.$ compact, $\left.i \in \mathbb{N}\right\}$ ), the acyclic maps $\mathbb{V}$ (with acyclic values), the O'Neill maps $\mathbb{N}$ (with values consisting of one or $m$ acyclic components, where $m$ is fixed), the approachable maps $\mathbb{A}$ in a topological vector space admissible maps in the sense of Gorniewicz, permissible maps of Dzedzej, and many others.

Remark 2.1. For a convex space $(X, D), X$ itself is not necessarily convex. To this end, we observe the following:

EXAMPLE 2.1. Let $X$ be any space containing an $n$-simplex $\Delta_{n}$ as a subspace and $D$ the set of vertices of $\Delta_{n}$. Then $(X, D)$ is a convex space. Note than $X$ may not be convex, but $D$-convex.

For a convex subset $X$ of a linear space $E$ and a topological space $Y$, let $S$, $T: X \rightarrow 2^{Y}$ be two set-valued mappings. If for each finite subset $A$ of $X$, we have $T($ co $A) \subseteq S A$, then $S$ is called generalised KKM mapping with respect to $T$.

REMARK 2.2. The notion of generalised KKM mapping was introduced by Park [6] and followed by others (see, also Chang and Yen [3]).

Let $(X, D)$ be a convex space and $Y$ a topological space. Let $S, T: X \rightarrow 2^{Y}$ be two set valued mappings. If for each $A \in\langle D\rangle$, we have $T(\operatorname{co} A) \subseteq S A$, then $S$ is called a $T_{D}$-KKM mapping with respect to $T$. Let $S$ be a $T_{D}$-KKM mapping and the family $\{S x: x \in D\}$ has the finite intersection properly, where $S x$ denotes the closure of $S x$. Then we say $T$ has the D-KKM property. Denote

$$
\operatorname{D-KKM}(X, Y)=\left\{T: X \rightarrow 2^{Y} \mid T \text { has D-KKM property }\right\} \text {. }
$$

When $X=D$, a convex subset of a linear space, the set-valued maping $S$ is called a generalised KKM, with respect to $T$, and $T$ is said to have the KKM property. In 
this case, we denote

$$
\mathrm{KKM}(X, Y)=\left\{T: X \rightarrow 2^{Y} \mid T \text { has KKM property }\right\}
$$

REMARKS 2.3. We remark that:

1. The notion of KKM property was introduced by Chang and Yen [3].

2. Our notion of D-KKM mapping is more general than the notion of generalised KKM mapping in the sense that we do not require $X$ to be a convex subset of a linear space.

3. The following example shows that the D-KKM property is weaker than the KKM property.

EXAMPLE 2.2. Let $D \in\{[0,1 / 2),(1 / 2,1]\}, X=[0,1] \subset R$, the set of reals $Y=[0,1]$, where both $R$ and $Y$ are endowed with Euclidean topology and let $S, T: X \rightarrow 2^{Y}$ be defined as follows:

then

$$
S x=T x= \begin{cases}\{0\}, & x \in\left[0, \frac{1}{2}\right), \\ \left\{0, \frac{1}{2}, 1\right\} & x=\frac{1}{2} \\ \{1\}, & x \in\left[\frac{1}{2}, 1\right),\end{cases}
$$

(i) $S$ and $T$ are closed (compact) for each $x \in X$;

(ii) $T$ is compact (hence upper semicontinuous);

(iii) $(X, D)$ is a convex space; and

(iv) $T(\operatorname{co} A) \subseteq S A$ for each $A \in\langle D\rangle$.

However, the family $(S x: x \in X\}$ has no finite intersection property and so $T \notin \mathrm{KKM}$. On the other hand, the family $\{S x: x \in D\}$ has finite intersection property. Hence $T \in \mathrm{D}-\mathrm{KKM}$.

Let $X$ and $Y$ be two topological spaces. $A$ subset $B$ of $Y$ is said to be compactly closed if for any compact subset $K$ of $Y, B \cap K$ is closed in $K$.

\section{Main Results}

We first, present the following proposition which characterises $T_{D}$-KKM mappings.

Proposition 3.1. Let $(X, D)$ be a convex space, $Y$ a set, and let $S: X$ $\rightarrow 2^{Y}, T: X \rightarrow 2^{Y}$ be two set-valued mappings. Then the following conditions are equivalent:

(i) $S$ is a $T_{D}$-KKM mapping.

(ii) $\cos S_{D}^{*} Y \subseteq T_{D}^{*} Y$ for each $y \in Y$.

(iii) The mapping co $T S^{*}$ has no fixed point in $Y$. 
Proof: (i) $\Leftrightarrow$ (ii): Suppose $S$ is a $T_{D}$-KKM mapping with respect to $T$. We now suppose that there exists $y \in Y$ such that $\operatorname{co} S_{D}^{*} y \not \subset T_{D}^{*} y$. Then there exists $a\left\{x_{1}, x_{2}, \ldots, x_{n}\right\} \in\langle D\rangle \subseteq S_{D}^{*} y$ and $x \in \operatorname{co}\left\{x_{1}, x_{2}, \ldots, x_{n}\right\}$ such that $x \notin T_{D}^{*} y$. This implies that $y \notin \bigcup_{i=1}^{n} S x_{i}$ and $y \in T x$. Hence

$$
T\left(\operatorname{co}\left\{x_{1}, x_{2}, \ldots, x_{n}\right\}\right) \not \subset[b] \bigcup_{i=1}^{N} S x_{i}
$$

which contradicts the assumption that $S$ is a $T_{D}$-KKM mapping.

Conversely, suppose that $\operatorname{co} S_{D}^{*} y \subseteq T_{D}^{*} y$ for each $y \in Y$. Then we show that $S$ is a $T_{D}$-KKM mapping. In doing so, we adopt contrapositive approach. So, let there be a set $A=\left\{x_{1}, x_{2}, \ldots, x_{n}\right\} \in\langle D\rangle$ such that $T(\operatorname{co} A) \not \subset S A$. Then there exists a $x \in \operatorname{co} A$ and $y \in T x$ such that $y \notin \bigcup_{i=1}^{n} S x_{i}$, that is, $x_{i} \in S_{D}^{*} Y$ for each $i=1,2, \ldots, n$ and hence $x \in \operatorname{co} S_{D}^{*} y$. But $y \in T x$, it folows that $x \notin T_{D}^{*} y$ which implies $\cos S_{D}^{*} y \not \subset T_{D}^{*} y$. This completes the proof.

(iii) $\Leftrightarrow$ (i): Suppose that $y \in \operatorname{co} T S^{*} y$ for some $y \in Y$, then there exist a finite set $\left\{x_{1}, x_{2}, \ldots, x_{n}\right\} \subseteq D$ and a finite set $\left\{y_{1}, y_{2}, \ldots, y_{n}\right\} \subseteq Y$ with $x_{i} \in S^{*} y$ and $y_{i} \in T x_{i}$, $I=1,2, \ldots, n$ such that $y=\sum_{i=1}^{n} \lambda_{i} y_{i}$, where $0<\lambda_{1}<1$ and $\sum_{i=1}^{n} \lambda_{i}=1$ which implies that $y \in T\left(\operatorname{co}\left(x_{1}, x_{2}, \ldots, x_{n}\right)\right)$ and $y \notin \bigcup_{i=1}^{n} S x_{i}$. Hence $S$ is not $T_{D}-\mathrm{KKM}$.

Conversely, suppose that $S$ is not a $T_{D}-\mathrm{KKM}$ mapping, then there exist $\left\{x_{1}, x_{2}\right.$, $\left.\ldots, x_{m}\right\} \subseteq D$ and $z \in T\left(\operatorname{co}\left\{x_{1}, x_{m}, \ldots, x_{m}\right\}\right)$ such that $z \notin \bigcup_{j=1}^{m} S x_{j}$. Since $z$ $\notin \bigcup_{j=1}^{m} S x_{j}$, it follows that $x \in \bigcup_{j=1}^{m}\left(Y \backslash S x_{j}\right)$. This implies $x_{j} \in S^{*} z$ for each $j=1,2, \ldots, m$ and $T x_{j} \subseteq T S^{*} z$. Hence $\operatorname{co}\left(\bigcup_{j=1}^{m} T x_{j}\right) \subseteq \operatorname{co} T S^{*} z$, and so $z \in \operatorname{co} T S^{*} z$. This completes the proof.

In our next proposition, we state some properties of the $T_{D}$-KKM family.

Proposition 3.2: Let $(X, D)$ be a convex space, and let $Y, Z$ be two topological spaces. Then

(i) $T \in D-\operatorname{KKM}(X, Y)$ if and only if $\left.T\right|_{\Delta} \in D-\operatorname{KKM}(\Delta Y)$ for each polytope $\Delta$ of $(X, D)$,

(ii) if $T \in D-\operatorname{KKM}(X, Y)$ and $f \in \mathbb{C}(Y, Z)$, then $f T \in D-\operatorname{KKM}(X, Z)$; and

(iii) if $Y$ is a normal space, $\Delta$ a polytope of $(X, D)$, and if $T: \Delta \rightarrow 2^{Y}$ is a set-valued mapping such that for each $f \in \mathbb{C}(Y, \Delta), f T$ has a fixed point in $\Delta$ then $T \in D-\operatorname{KKM}(Y, \Delta)$. 
Proof: (i) is obvious. To prove (ii), let $S: X \rightarrow 2^{Z}$ be a $f T_{D}$-KKM mapping such that $S x$ is closed for each $x \in D$. Then, for a set $\left\{x_{1}, x_{2}, \ldots, x_{n}\right\} \in\langle D\rangle$, since $S$ is a $D$-KKM mapping with respect to $f T$, we have

$$
f T\left(\operatorname{co}\left\{x_{1}, x_{2}, \ldots, x_{n}\right\}\right) \subset \bigcup_{i=1}^{n} S x_{i}
$$

which implies that

$$
T\left(\operatorname{co}\left\{x_{1}, x_{2}, \ldots, x_{n}\right\}\right) \subseteq f^{1}\left(\bigcup_{i=1}^{n} S x_{i}\right)=\bigcup_{i=1}^{n} f^{1} S x_{i} .
$$

Therefore, $f^{-1} S$ is a $T_{D}$-KKM mapping. By hypothesis, $T \in D$-KKM $(X, Y)$ and hence the family $\left\{f^{-1} S x: x \in D\right\}$ has finite intersection property which implis that $\left\{\left(f^{-1} S x\right): x \in D\right\}$ has finite intersection property. This shows that $f T \in D-\operatorname{KKM}(X, Z)$ and we complete the proof of (ii).

To prove (iii), let $S: \Delta \rightarrow 2^{Y}$ be a $T_{D}$-KKM mapping such that $S x$ is closed for each $x \in \Delta$. Suppose, if possible, that the family $\{S x: x \in \Delta\}$ has no finite intersection property, then the exists a finite subset $\left\{x_{1}, x_{2}, \ldots, x_{n}\right\}$ of $\Delta$ such that $\bigcup_{i=1}^{n} S x_{i}=\emptyset$, and so $\bigcap_{i=1}^{n}\left(Y \backslash S x_{j}\right)=Y$.

Let $\left\{\alpha_{i}\right\}_{i=1}^{n}$ be a partition of unity subordinated to $\left\{Y \backslash S x_{i}\right\}_{i=1}^{n}$ such that each $\alpha_{i}$ has its support in some $Y \backslash S x_{i}(i=1,2, \ldots, n)$, and let $\Delta^{\prime}=\operatorname{co}\left\{x_{1}, x_{2}, \ldots, x_{n}\right\}$. Define the mapping $f: Y \rightarrow \Delta^{\prime}$ by

$$
f(y)=\sum_{i=1}^{n} \alpha_{i}(y) x_{i}, \text { for each } y \in Y .
$$

Then $f \in C(Y, \Delta)$. Hence, by our assumption, there exists $x \in \Delta$ such that $x$ $\in f T x$; that is, there exists $\xi \in T x$ such that $x=f(\xi) \sum_{i=1}^{n} \alpha_{i}(\xi) x_{i}$. Suppose $I(\xi)=\{i$ $\left.\in\{1,2, \ldots, n\}: \alpha_{i}(\xi)>0\right\}$. Since $\alpha_{i}(\xi)>0$ implies $\xi \notin S x_{i}, i=1,2, \ldots, n$, and so $\xi \notin T\left(\Delta^{\prime}\right)$. This implies that $T \notin D$-KKM $(X, Y)$ and we complete the proof of (ii). $\square$

This theorem generalises the well-known Fan-KKM Theorem [4, Theorem 3].

Theorem 3.3. Let $(X, D)$ be a convex space, $Y$ a topological space, let $t$ : $X \rightarrow Y$ be a single-valued mapping, $S: X \rightarrow 2^{Y}$ a set-valued mapping satisfying the conditions:

(i) $S$ is $t_{D}-K K M$ mapping.

(ii) For each $x \in D, S x$ is compactly closed in $Y$; and

(iii) there exists a non empty compact convex subset $D_{0}$ of $D$ and a compact subset $K$ of $Y$ such that $\bigcap_{x \in D_{0}} S x \subseteq K$. 
Then $\bigcap_{x \in D} S x \neq \emptyset$.

Proof: Suppose, on the contrary, that $\bigcap_{x \in D} S x=\emptyset$ then $\bigcup_{x \in D}(Y \backslash S x)=Y$. It follows that $\bigcup_{x \in D}((Y \backslash S x) \cap K)=K$. Since $K$ is compact and $Y \backslash S x$ is compactly open in $Y$ for each $x \in D$, there exists a finite set $\left\{x_{1}, x_{2}, \ldots, x_{n}\right\} \in\langle D\rangle$ such that

$$
\bigcup_{i=1}^{n}\left(\left(Y \backslash S x_{i}\right) \cap K\right)=K \text {. }
$$

Let $t x_{i}=y_{i}$ for each $i \in\{1,2, \ldots, n\}$. Now, we suppose that $D_{1}=\operatorname{co}\left(D_{0}\right.$ $\left.\cup\left\{y_{1}, y_{2}, \ldots, y_{n}\right\}\right)$. Then $D_{1}$ is a compact convex subset of $Y$. By $\left({ }^{*}\right)$ and (iii), we have $\bigcup_{t x \in D_{1}}(Y \backslash S x)=Y$ which implies that $\cup(Y \backslash S x) \cap D_{1}=D_{1}$. By compactness of $D_{1}$ and (ii) there exist a finite subset $\left\{z_{1}, z_{2}, \ldots, z_{m}\right\} t x \in D_{1}$ of $X$ such that $w_{j}=t z_{j} \in D_{1}$ for each $j \in\{1,2, \ldots, m\}$ and $\cup\left(Y \backslash S x_{j}\right) \bigcap_{j=1}^{m} D_{1}=D_{1}$. Let $\left\{\alpha_{j}\right\}_{j=1}^{m}$ be a patition of unity subordinate to $\left\{\left(Y \backslash S x_{j}\right) \cap D_{1}\right\}_{j=1}^{m}$ and let $\Delta^{*}=\operatorname{co}\left\{w_{1}, w_{2}, \ldots, w_{m}\right\}$. We now define the function $f: \Delta^{*} \rightarrow \Delta^{*}$ by

$$
f(y)=\sum_{j=1}^{m} \alpha_{j}(y) w_{j}\left(y \in \Delta^{*}\right) .
$$

Clearly $f$ is a continuous function and so it has fixed point in $\Delta^{*}$, that is, there is a $y^{*} \in \Delta^{*}$ such that $f\left(y^{*}\right)=y^{*}$.

Let $J\left(y^{*}\right)=\left\{j \in\{1,2, \ldots, m\}: \alpha_{j}\left(y^{*}\right)>0\right\}$. Since $\alpha_{j}\left(y^{*}\right)>0$ implies $y^{*}$ $\in Y \backslash S z_{j}$, it follows that for each a $j \in J\left(y^{*}\right), Y^{*} \notin S z_{j}$. Hence

$$
y^{*}=\sum_{j \in J\left(y^{*}\right)} \alpha_{j}\left(y^{*}\right) w_{j} \notin \bigcup_{j \in J\left(y^{*}\right)} S z_{j},
$$

a contradiction to assumption (i). This completes the proof.

REMARK 3.1. We remark that:

(1) For the case $D=X$, Theorem 3.3 reduces to Theorem 2 of Chang and Yen [3].

(2) In the case $X$ is a convex subset of $Y, D=X$ and $t$ is the inclusion mapping, then Theorem 3.3 reduces to Fan-KKM Theorem [4, Theorem 3].

It may be observed that many others had defined several families of set valued mappings such as, $\mathbf{M}(X, Y)(\operatorname{see}[\mathbf{1 0}]) \mathbb{K}_{C}(X, Y)$ (see [7]), $\mathbf{V}_{C}(X, Y)$ (see [11]), $\mathbf{A}_{C}(X, Y)$ 
(see [1]) and $\mathfrak{U}_{C}^{K}(X, Y)$ (see[10]). It can be easily proved that the above families are contained in D-KKM $(X, Y)$ by the above proposition, and so we omit the proof. We have an interesting example (Example 3.1 below) which shows that the D-KKM family is indeed larger than the others.

EXAmple 3.1. Let $D \in\{[-1,0),(0,1]\}, X=[-1,1] \subset R$ and $Y=[-1,1]$, where $R$ and $Y$ both are endowed with the Euclidean topology and let $S=T: X \rightarrow 2^{Y}$ be defined as follows:

$$
T x= \begin{cases}\left\{\sin \frac{1}{x^{2}}\right\}, & x \in[-1,0), \\ \{0\}, & x \in 0, \\ \left\{\sin \frac{1}{x}\right\}, & x=(0,1] .\end{cases}
$$

\section{SOME APPLICATIONS}

We now apply the main results of Section 3 to get some existence theorems for maxmal elements of a binary relation, price equilibria, and generalised variational inequalities.

Let $D$ be a set, and let $\sim$ be a binary relation on $D$. We call $y^{*}\left(y^{*} \in D\right)$ a maximal element of $D$ (with respect to binary relation $\sim$ ) if $x \sim y^{*}$ for all $x \in D$.

We now use Theorem 3.3 to obtain the following theorem.

THEOREM 4.1. Let $(X, D)$ be a convex space, $\sim$ be a binary relation defined on $D$, and let $t \in D-K K M(X, X)$ satisfy the following conditions:

(i) for each $x \in X, x \notin \operatorname{co}\{y \in D: y \sim x$ for some $y=t x\}$,

(ii) for each $x \in X$, the set $\{y \in D: y \sim x\}$ is compactly closed in $X$, and

(iii) there exists a nonempty compact convex subset $D_{0}$ of $D$ and a compact subset $K$ of $X$ such that for each $y \in X \backslash K$, there is $x \in D_{0}$ with $x \sim y$.

Then $\sim$ has a maximal element on $D$.

Proof: Define $S: X \rightarrow 2^{X}$ by

$$
S y=\{y \in D: y \sim x\}(x \in X) .
$$

Then, by assumption (ii), for each $x \in X, S x$ is compactly closed in $X$. By assumption (i) for each $x \in X, x \notin \cot S^{*} x$, hence, by and Proposition 3.1, $S$ is a $t_{D}-\mathrm{KKM}$ mapping. Moreover, condition (iii) implies that $\bigcap_{x \in D} S x \subseteq K$. Hence, by Theorem 3.3, we have

$$
\bigcap_{x \in D} S x \neq \emptyset
$$

Suppose $y^{*} \in \bigcap_{x \in D} S x$ then $x \sim y^{*}$ for all $x \in D$. It follows, therefore, that $y^{*}$ is a maximal element on $D$. 
This completes the proof.

For the case that $t$ is a single-valued continuous mapping we have the following:

Corollary 4.2. Let $(X, D)$ be a convex space, $\sim$ be a binary relation defined on $D$, and let $t \in \mathbb{C}(X, X)$. If the conditions (i) and (ii) of Theorem 4.1 hold, and if for each $x \in X, x \notin \operatorname{co}\{y \in D: y \sim s x, y=t x\}$ then $\sim$ has a maximal element on $D$.

REMARK 4.1. For the case $D=X=D_{0}=K$ is a compact convex subset of a topological vector space and $t$ is the identity mapping on $X$, our Corollary 4.2 reduces to Theorem 5.1 of Yannelis and Prabhakar [14].

In the sequel, let $E$ denote a topolgical vector space, $E^{*}$ the dual space of $E$, and let $(u, v)$ denote the pairing between $u \in E$ and $v \in E^{*}$.

We now apply Theorem 4.1, to obtain the following theorem concerning general variational inequalities of Yao type [13].

CoRollary 4.3. Let $X$ be a nonempty subset of $E$, let $(X, D)$ be a convex space, let $t \in D-K K M(X, X)$, and let $g: X \rightarrow X, f: X \rightarrow E^{*}$ be two single-valued functions satisfying the following conditions:

(i) For each $x \in X, x \notin \operatorname{co}\{y \in D: \sup \langle g(y)-g(z), f(z)\rangle>0, y=t x\}$,

(ii) For each $x \in X$, the set $\{y \in D:\langle g(x)-g(y), f(y)\rangle \leqslant 0\}$ is a compactly closed subset of $X$, and

(iii) There exists a nonempty compact convex subset $D_{0}$ of $D$, and a compact subset $K$ of $X$ such that for each $y \in X \backslash K$, there is $x_{0} \in t^{-1}\left(D_{0}\right)$ with $\langle g(x)-g(y), f(y)\rangle>0$. Then there exists $y^{*} \in X$ such that

$$
\left\langle g(x)-g\left(y^{*}\right), f\left(y^{*}\right)\right\rangle \leqslant 0 \text { for all } x \in D .
$$

Proof: Define a binary relation $\sim$ on $D$ by $y \sim x$ if $\langle g(y), f(x)\rangle>\langle g(x), f(x)\rangle$, $x, y \in D$. Then it is easy to see that all conditions of Theorem 4.1 are satisfied. Hence there exists $y^{*} \in D$ such that $x \sim y^{*}$ for all $x \in D$. Hence, we have

$$
\left\langle g(x), f\left(y^{*}\right)\right\rangle \leqslant\left\langle g\left(y^{*}\right), f\left(y^{*}\right)\right\rangle, \text { for all } x \in D .
$$

For the case $g:=i d_{X}$ (the identity mapping on $X$ ), we have the following:

Corollary 4.4. Let $(X, D)$ be defined as in Corollary 4.3. Let $t \in D$ $\operatorname{KKM}(X, X)$ and let $f: X \rightarrow E^{*}$ be a function. Let condition (i) of Corollary 4.3 hold, and let the following conditions hold:

(ii) for each $x \in D, x \notin \operatorname{co}\{y \in X: \sup \langle y-z, f(z)\rangle>0, y \in t x\}$,

(iii) for each $x \in D$, the set $\{y \in X:\langle x-y, f(y)\rangle \leqslant 0\}$ is a compactly closed subset of $X$, and

(iv) there exists a nonempty compact convex subset $D_{0}$ of $D$, and a compact subset of $K$ of $X$ such that for each $y \in X \backslash K$, there is $x_{0} \in t^{-1}\left(D_{0}\right)$ such that $\langle x-y, f(y)\rangle>0$. 
Then there exists $y^{*} \in D$ such that

$$
\left\langle x-y^{*}, f\left(y^{*}\right)\right\rangle \leqslant 0 \text { for all } x \in D
$$

Remark 4.2. For the case $D=X$, our Corollary 4.4 reduces to Corollary 6 of Chang and Yen [3]. For the case $t$ is a single-valued continuous function, we have the following:

Corollary 4.5. Let $(X, D)$ be as defined in Theorem 4.1. Let $T \in C(X, X)$, and let $f: X \rightarrow E^{*}$ be a function such that conditions (iii), (iv) of Corollary 4.4 hold. If for each $x \in D, x \notin \operatorname{co}\{y \in X:\langle y-t x, f(s x)\rangle>0\}$, then there exists $y^{*} \in D$ such that

$$
\left\langle x-y^{*}, f\left(y^{*}\right)\right\rangle \leqslant 0 \text { for all } x \in D .
$$

In the sequel, we denote by $D \subseteq X$ a closed convex cone in $E$. We now present the following corollary concerning the existence of price equilibria (see, also Border [2, p. 39], Chang and Yen [3, Corollary 7] and Tian [13]).

Corollary 4.6. Let $(X, D)$ be a convex space, $D$ a closed convex cone in $E$ let $t \in D-K K M(X, X)$, and let $f: X \rightarrow E^{*}$ be a function such that the assumption (i) (iv) of Corollary 4.4 hold. Then there exists $y^{*} \in X$ such that

$$
\left\langle x, f\left(y^{*}\right)\right\rangle \leqslant 0 \text { for all } x \in D \text { and }\left\langle y^{*}, f\left(y^{*}\right)\right\rangle=0 \text {. }
$$

Proof: By Corollary 4.4, there exists $y^{*} \in D$ such that

$$
\left\langle x-y^{*}, f\left(y^{*}\right)\right\rangle \leqslant 0 \text { for all } x \in D \text {. }
$$

With any loss of generality, we may assume that $0 \in D$. So, taking $x=0$ in the above inequality yields $\left\langle y^{*}, f\left(y^{*}\right)\right\rangle \geqslant 0$, and setting $x=2 y^{*}$ yields $\left\langle y^{*}, f\left(y^{*}\right)\right\rangle \leqslant 0$. Thus $\left\langle y^{*}, f\left(y^{*}\right)\right\rangle=0$. Hence

$$
\left\langle x, F\left(y^{*}\right)\right\rangle \leqslant 0 \text { for all } x \in D
$$

REMARK 4.3. For the case that $D=X$, on Corollary 4.6 reduces to Corollary 7 of Chang and Yen [3].

\section{REFERENCES}

[1] H. Ben-El-Mixhaiekh and P. Dequire, 'Approachability and fixed points for non-convex set valued maps', J. Math. Anal. Appl. 170 (1992), 477-500.

[2] K.C. Border, Fixed point theorems with applications to economics and game theory (Cambridge University Press, Cambridge, 1989).

[3] T.H. Chang, 'Generalized KKM theorm and its application', Far East J. Math 4 (1996), 137-147. 
[4] K. Fan, 'Some properties of convex sets related fixed point theorems', Math. Ann. 26 (1984), 519-537.

[5] M. Lassoude, 'Fixed point Kakutani factorizable multifunctions', J. Math. Anal. Appl. $152(1990), 46-60$.

[6] S. Park, 'Generalizations of Ky Fan's matching theorems and their application', J. Math. Anal. Appl. 141 (1989), 164-176.

[7] S. Park, 'Convex spaces and KKM families of subsets', Bull. Korean Math. Soc. 27 (1990), 11-14.

[8] S. Park, 'Foundations of KKM theory via coincidence of composites of upper semi continuous maps', J. Korean Math. Soc. 31 (1994), 493-519.

[9] S. Park, S.P. Singh and B. Watson, 'Some fixed point theorems for composite of a cyclic maps', Proc. Amer. Math. Soc. 121 (1994), 1151-1158.

[10] N. Shioji, 'A further generalization of the Knaster-Kuratowski-Mazur Kiewicz Theorem', Proc. Amer. Math. Soc. 111 (1991), 187-195.

[11] G.Q. Tian, 'Generalized KKM theorem, mini-max inequalities, and their application', $J$. Optim. Theory Appl. 83 (1994), 375-389.

[12] N.C. Yannelis and N.D. Prabhakar, 'Existence of maximal elements and equilibria in linear topological spaces', J. Math. Econ. 12 (1983), 233-245.

[13] J.C. Yao, 'On the general variational inequality', J. Math. Anal. Appl. 174 (1993), 550-555.

Department of Mathematics

Kalyan Mahavidyalaya

Bhilai Nagar (C.G.)

490006 India
Department of Mathematics and Statistics

College of Science

Sultan Qaboos University

Al-Khod, Muscat

Sultanate of Oman 